\title{
Update on the critical endpoint of the finite temperature phase transition for three flavor QCD with clover type fermions
}

\author{
Yoshifumi Nakamura*a,b, Xiao-Yong Jin ${ }^{a \dagger}$, Yoshinobu Kuramashi ${ }^{c, d, a}$, \\ Shinji Takeda ${ }^{e, a}$, Akira Ukawa ${ }^{a}$ \\ ${ }^{a}$ RIKEN Advanced Institute for Computational Science, Kobe, Hyogo 650-0047, Japan \\ ${ }^{b}$ Graduate School of System Informatics, Department of Computational Sciences, Kobe \\ University, Kobe, Hyogo 657-8501, Japan \\ ${ }^{c}$ Faculty of Pure and Applied Sciences, University of Tsukuba, Tsukuba, Ibaraki 305-8571, \\ Japan \\ ${ }^{d}$ Center for Computational Sciences, University of Tsukuba, Tsukuba, Ibaraki 305-8577, Japan \\ ${ }^{e}$ Institute of Physics, Kanazawa University, Kanazawa 920-1192, Japan \\ E-mail: nakamura@riken.jp
}

\begin{abstract}
We presented "Critical endpoint of finite temperature phase transition for three flavor QCD", results for the critical endpoint of finite temperature phase transition of $N_{f}=3$ QCD at zero chemical potential. We employ the renormalization-group improved Iwasaki gauge action and non-perturbatively $O(a)$-improved Wilson-clover fermion action. The critical endpoint is determined by using the intersection point of kurtosis for the temporal size $N_{t}=4,6,8$. Spatial sizes of $N_{l}=6-16\left(N_{t}=4\right), 10-24\left(N_{t}=6\right)$, and 12-24 $\left(N_{t}=8\right)$ are employed.
\end{abstract}

\section{Reference:}

Xiao-Yong Jin, Yoshinobu Kuramashi, Yoshifumi Nakamura, Shinji Takeda, Akira Ukawa, Critical endpoint of finite temperature phase transition for three flavor QCD,

Phys. Rev. D in press. [arXiv:1411.7461].

The 32nd International Symposium on Lattice Field Theory

23-28 June, 2013

Columbia University New York, NY

\footnotetext{
* Speaker.

${ }^{\dagger}$ Present address: Argonne Leadership Computing Facility, Argonne National Laboratory, Argonne, Illinois 60439, USA
} 